\title{
Tight generalization guarantees for the sampling and discarding approach to scenario optimization
}

\author{
Licio Romao, Kostas Margellos and Antonis Papachristodoulou
}

\begin{abstract}
We consider the scenario approach to deal with convex optimization programs affected by uncertainty, which is in turn represented by means of scenarios. An approach to deal with such programs while trading feasibility to performance is known as sampling and discarding in the scenario approach literature. Existing bounds on the probability of constraint satisfaction for such programs are not tight. In this paper we use learning theoretic concepts based on the notion of compression to show that for a particular class of convex scenario programs, namely, the so called fully-supported ones, and under a particular scenario discarding scheme, a tight bound can be obtained. We illustrate our developments by means of an example that admits an analytic solution.
\end{abstract}

\section{INTRODUCTION}

The scenario approach theory [1]-[3] is a successful randomization technique to solve convex uncertain optimization problems. It is based on representing uncertainty by means of scenarios, accompanying the optimal solution of the resulting scenario program with a priori certificates on the probability of constraint violation. The scenario approach theory has been introduced in [1] and has undergone several developments, including tightness of the bound on the probability of constraint violation [2], the sampling and discarding [4] or constraint removal scheme [5] to trade feasibility to performance, extension to non-convex programs [6], and, more recently, a posteriori assessing the scenario solution [7]-[10] as well as game theoretic extensions [11]-[13].

This paper is related to the a priori results of the scenario approach theory. In particular, it is shown in [2] that a computationally efficient bound on the probability of constraint violation can be obtained. However, the cost associated to the optimal solution of the resulting scenario program might be high, as the bound on the probability of constraint violation is conservative for the general case and tight only for the class of fully-supported programs. To mitigate this inherent conservatism, [4], [5] have developed a strategy that allows the scenario user to discard some of the original scenarios in order to enhance performance. This strategy was introduced in [4] and was termed as sampling and discarding approach, as well as in [5] where it was referred to as scenario approach with constraint removal. The sampling and discarding strategy offers a way to trade two conflicting objectives: the probability of constraint violation and perfor-

L. Romao is supported by the Coordination for the Improvement of Higher Education Personnel (CAPES) - Brazil. The work of K. Margellos and A. Papachristodoulou has been supported by EPSRC UK under grants $\mathrm{EP} / \mathrm{P} 03277 \mathrm{X} / 1$ and $\mathrm{EP} / \mathrm{M} 002454 / 1$, respectively.

The authors are with the Department of Engineering Science, University of Oxford, Parks Road, Oxford OX1 3PJ, UK. \{1 icio.romao, kostas.margellos, antonis\}eeng.ox.ac.uk. mance. However, as opposed to the tight bound of [2], the bound in [4] is not tight. In fact, it is shown in [4] that there exists an instance of a particular combinatorial constraint removal scheme that yields a solution that possesses a tighter bound on the probability of constraint violation; however, the presented argument is not constructive.

In this paper, we revisit the sampling and discarding framework and prove two main results for the class of fullysupported problems: (1) We show that a tighter bound on the probability of constraint violation can be obtained by considering a removal scheme that consists of a cascade of scenario programs, where at each stage the support set, a concept at the core of the scenario approach theory that will be formalized in the sequel, associated to the optimal solution is removed. To establish this tighter bound we analyze the constraint violation properties of the resulting solution within a probably approximately correct (PAC) [14] learning framework based on the notion of compression [15], [16]. (2) Similarly to [2], we show that the proposed bound on the probability of constraint violation is tight by characterizing a class of optimization problems that achieves the proposed bound with equality. It appears that the proposed bound is more general, and albeit not tight, is valid for any convex scenario program under a mild non-degeneracy assumption. This extension can be found in [17].

Section II provides some background concepts from the scenario approach and learning theoretic literature. Section III introduces the proposed discarding scheme and states our main result. Section IV shows that the proposed result is tight for a certain class of programs, and illustrates this by means of an example that admits an analytic solution. Finally, Section V concludes the paper and provides some directions for future work. All omitted proofs can be found in [17].

\section{BACKGROUND KNOWLEDGE}

\section{A. The scenario theory}

Let $(\Delta, \mathcal{F}, \mathbb{P})$ be a probability measure space, where $\Delta$ is the domain in which random variables is defined, $\mathcal{F}$ is a $\sigma$ algebra of subsets of $\Delta$, and $\mathbb{P}: \Delta \rightarrow[0,1]$ is a probability measure on $\Delta$. We assume that the probability $\mathbb{P}$ is timeinvariant and unknown; moreover, we suppose independent samples from $\mathbb{P}$ are available. For a fixed $m \in \mathbb{N}$, we denote these samples as $S=\left(\delta_{1}, \ldots, \delta_{m}\right)$, and refer to each element $\delta_{i} \in S$ as a scenario. Note that $S$ can be seen as an element in the product space $\Delta^{m}=\prod_{i=1}^{m} \Delta$ and is distributed according to $\mathbb{P}^{m}$, due to the fact that $\delta_{i}$ 's, $i=1, \ldots, m$, are assumed to be independent and identically distributed (i.i.d.). In this context, we can associate a useful probability 
space, namely, $\left(\Delta^{m}, \otimes \mathcal{F}, \mathbb{P}^{m}\right)$, where $\otimes \mathcal{F}$ is the smallest $\sigma$-algebra containing the cones sets $\prod_{i=1}^{m} \mathcal{F}$.

Given $m \in \mathbb{N}$, let $S=\left(\delta_{1}, \ldots, \delta_{m}\right)$ be a collection of $m$ i.i.d. samples as explained in the previous paragraph, and consider the optimization problem

$$
\begin{aligned}
\underset{x \in X}{\operatorname{minimize}} & c^{\top} x \\
\text { subject to } & g(x, \delta) \leq 0, \text { for all } \delta \in S,
\end{aligned}
$$

where $x \in \mathbb{R}^{d}$ is the optimization variable, $c \in \mathbb{R}^{d}$ is a given vector representing the objective function, $g: \mathbb{R}^{d} \times \Delta \rightarrow \mathbb{R}$ is convex in $x$ for all $\delta \in S$, and $X \subset \mathbb{R}^{d}$ is a closed convex set. Note that the objective function in (1) can be considered linear without loss of generality, as otherwise one may use an epigraph formulation to recast any convex problem into the form of (1).

Assumption 1: For each $m \in \mathbb{N}$, the solution of the optimization problem (1) exists and is unique.

The uniqueness requirement is a mild assumption in most applications and can be ensured by a tie-break rule procedure that selects one solution among all the elements in the optimal set of (1). Similarly, existence can be ensured by standard techniques in variational methods, for instance, the optimal set of (1) is non-empty if we assume that there exists a $\gamma \in \mathbb{R}$ such that the set $X \cap\left\{x \in \mathbb{R}: c^{\top} x \leq \gamma\right\}$ is compact or if $X$ is compact.

We refer to problem (1) as a scenario program, as one constraint is enforced for each scenario in $S$. Let $x^{\star}(S)$ be the optimal solution of (1), where the argument aims to emphasize the dependence on the original samples given by $S$. One of the main results of the scenario theory is quantifying the probability of constraint violation associated to $x^{\star}(S)$.

Theorem 1 (Theorem 1, [2]): Consider Assumption 1, and fix $\epsilon \in(0,1)$. Let $m \in \mathbb{N}$ be given and denote by $x^{\star}(S)$ the optimal solution of (1). Then we have that

$$
\begin{gathered}
\mathbb{P}^{m}\left\{\left(\delta_{1}, \ldots, \delta_{m}\right) \in \Delta^{m}: \mathbb{P}\left\{\delta: g\left(x^{\star}(S), \delta\right)>0\right\}>\epsilon\right\} \\
\leq \sum_{i=0}^{d-1}\left(\begin{array}{c}
m \\
i
\end{array}\right) \epsilon^{i}(1-\epsilon)^{m-i} .
\end{gathered}
$$

The left-hand side of Theorem 1 is composed of two nested probabilities: the outer one represents the confidence with which the bound is valid, and the inner one stands for the risk incurred by the optimal solution of problem (1).

Definition 1 (Support scenario and support set): An element $\delta \in S=\left(\delta_{1}, \ldots, \delta_{m}\right)$ is called a support scenario if its removal changes the optimal solution $x^{\star}(S)$ of problem (1). The support set is the collection of all support scenarios and is denoted by $\operatorname{supp}\left(x^{\star}(S)\right)$.
Definition 2 (Fully supported problems): A scenario program is called fully supported if, with $\mathbb{P}^{m}$ probability one, the cardinality of the support set is equal to $d$.

An important feature of the bound in (2) is the fact that it holds for all convex optimization problems and all distributions $\mathbb{P}$. In fact, [2] showed that the class of fully-supported optimization problems achieves the bound of Theorem 1. In this sense, the bound in (2) is said to be tight (see [1]-[3] for more details).

\section{B. The sampling and discarding approach}

Let $m \in \mathbb{N}$ and $r<m$ be given, and consider the following optimization problem

$$
\begin{aligned}
\underset{x \in X}{\operatorname{minimize}} & c^{\top} x \\
\text { subject to } & g(x, \delta) \leq 0, \text { for all } \delta \in S \backslash R,
\end{aligned}
$$

where $x, c, X$ and $S$ are as in (1), and $R$ is a subset of the original sample set with cardinality equal to $r$. The main goal of the formulation (3) is to allow discarding some of the original constraints, thus leading to an improvement in terms of objective value. The sampling and discarding approach was introduced in [4], and has also been studied in [5] where it is referred to as scenario approach with constraint removal. It quantifies the probability of violation for the optimal solution (under Assumption 1) of (3). Let $x^{\star}(S)$ be the optimal solution of (3). We then have the following result.

Theorem 2 (Theorem 2.1, [4]): Consider Assumption 1, and fix $\epsilon \in(0,1)$. Let $m>d+r$ and denote by $x^{\star}(S)$ the optimal solution of (3). If all removed scenarios are violated by the resulting solution $x^{\star}(S)$, i.e., $g\left(x^{\star}(S), \delta\right)>0$ for all $\delta \in R$, with $\mathbb{P}^{m}$-probability one, then

$$
\begin{gathered}
\mathbb{P}^{m}\left\{\left(\delta_{1}, \ldots, \delta_{m}\right) \in \Delta^{m}: \mathbb{P}\left\{\delta: g\left(x^{\star}(S), \delta\right)>0\right\}>\epsilon\right\} \\
\leq\left(\begin{array}{c}
r+d-1 \\
r
\end{array}\right) \sum_{i=0}^{r+d-1}\left(\begin{array}{c}
m \\
i
\end{array}\right) \epsilon^{i}(1-\epsilon)^{m-i} .
\end{gathered}
$$

As opposed to the tight bound of Theorem 1, the bound of Theorem 2 is not tight. Indeed, in Section 4.2 of [4], the authors show that there exists a class of convex optimization programs and a discarding scheme such that the right-hand side of (4) can be replaced by $\sum_{i=0}^{r+d-1}\left(\begin{array}{c}m \\ i\end{array}\right) \epsilon^{i}(1-\epsilon)^{m-i}$; however, the argument is not constructive and is limited to an existential statement both as far as the discarding scheme and the problem class are concerned. This raises the following natural question: can one provide tight bounds for a scenario program with discarded constraints? In this paper, we answer the last question by providing a class of optimization problems and a scenario discarding scheme for which this is the case. Our analysis is based on compression learning concepts within the realm of PAC learnability. 


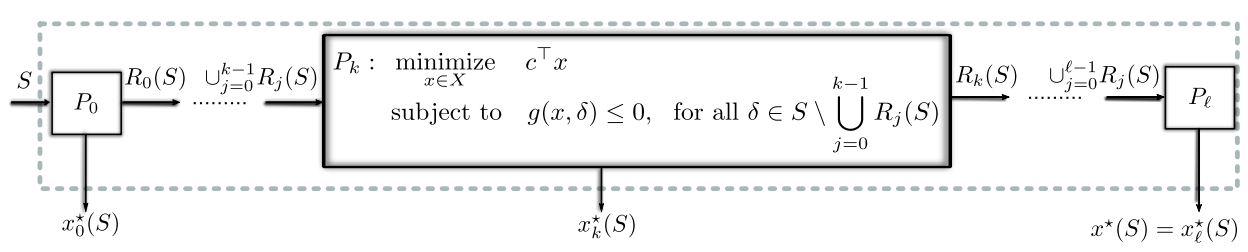

Fig. 1: Block diagram of the proposed scheme. For a given set of scenarios $S=\left\{\delta_{1}, \ldots, \delta_{m}\right\}$ with $(\ell+1) d<m$, we solve a cascade of $\ell+1$ optimization programs denoted by $P_{k}, k \in\{0, \ldots, \ell\}$. For each $k, k \in\{0, \ldots, \ell-1\}$, we remove $R_{k}(S)$ scenarios with $\left|R_{k}(S)\right|=d$ as in (6), hence, in total $r=\ell d$ scenarios (the ones in $\bigcup_{j=0}^{\ell-1} R_{j}(S)$ ) are discarded. The choice of each set of discarded scenarios depends on the initial set $S$, thus we introduce it as argument of $R_{k}$. The final solution is denoted by $x^{\star}(S)=x_{\ell}^{\star}(S)$.

\section{Learning theoretic background}

The main results of this paper are based on some concepts associated to the learning literature and on the interpretation of scenario theory within this framework given in [15].

Definition 3 (Compression set): Given $m \in \mathbb{N}$, and let $S=$ $\left(\delta_{1}, \ldots, \delta_{m}\right)$ be an element of $\Delta^{m}$. Define a mapping $\mathcal{A}$ : $\Delta^{m} \rightarrow 2^{\Delta}$. A subset $C \subset S$, with cardinality equal to $\zeta$, is said to be a compression of cardinality $\zeta$ for $\mathcal{A}$ if $\delta \in$ $\mathcal{A}(C)$, for all $\delta \in S$.

Roughly speaking, a subset $C$ is a compression for the mapping $\mathcal{A}$ if it assigns the same label to all scenarios in $S$ as if all the samples in $S$ were used. In the statistical learning theory this property is also known as consistency of $\mathcal{A}(C)$ with respect to the samples [14], [15]. The notion of compression set is crucial to characterize generalization properties of the mapping under unseen scenarios. In fact, after adapting the notation of paper [15] to the one of this paper, we can produce the following result.

Theorem 3 (Theorem 3, [15]): Fix $\epsilon \in(0,1)$ and $\zeta<m$. If there exists a unique compression set $C$ of cardinality $\zeta$, then

$$
\begin{aligned}
\mathbb{P}^{m}\left\{\left(\delta_{1}, \ldots, \delta_{m}\right) \in \Delta^{m}\right. & : \mathbb{P}\{\delta: \delta \notin \mathcal{A}(C)\}>\epsilon\} \\
& =\sum_{i=0}^{\zeta-1}\left(\begin{array}{c}
m \\
i
\end{array}\right) \epsilon^{i}(1-\epsilon)^{m-i}
\end{aligned}
$$

Note that for a fixed $\epsilon \in(0,1)$ the right-hand side of (5) tends to zero as $m$ tends to infinity. This property allows the user to quantify the number of samples that are needed to achieve a pre-specified confidence level, thus characterizing the non-asymptotic behaviour of the generalization properties associated to the mapping $\mathcal{A}$. In other words, Theorem 3 states that the mapping $\mathcal{A}$ is at least $(1-\epsilon)$-accurate as an approximation of $\Delta$ (approximately correct), with confidence (probably) equal to $1-\sum_{i=0}^{\zeta-1}\left(\begin{array}{c}m \\ i\end{array}\right) \epsilon^{i}(1-\epsilon)^{m-i}$.

\section{THE CASE OF FULLY-SUPPORTED SCENARIO PROGRAMS}

\section{A. The proposed removal scheme}

In this section we present the proposed removal scheme for the sampling and discarding framework. To this end, let $m \in \mathbb{N}$ and suppose $m$ i.i.d. samples from the unknown distribution $\mathbb{P}$ are available. Let $d$ be the dimension of the given optimization problem. Fix $r=\ell d$ and, for all $k \in\{1, \ldots, \ell\}$, consider

$$
\begin{aligned}
P_{k}: & \underset{x \in X}{\operatorname{minimize}} c^{\top} x \\
& \text { subject to } g(x, \delta) \leq 0, \text { for all } \delta \in S \backslash \bigcup_{j=0}^{k-1} R_{j}(S),
\end{aligned}
$$

where, for $j \in\{0, \ldots, \ell-1\}, R_{j}(S)$ represents a subset of $S$ with cardinality $d$, consisting of the removed scenarios at the $j$-th stage, and $c, X$, and $g: \mathbb{R}^{d} \times \Delta \rightarrow \mathbb{R}$ are defined as in (1) and (3). For $k=0$, we define $P_{0}$ as in (1), i.e., it is a scenario optimization problem where all the scenarios are enforced.

Assumption 2: We assume that, for all $k \in\{0, \ldots, \ell\}$,

i) The solution of $P_{k}$ exists and is unique with $\mathbb{P}^{m}$ probability one;

ii) $P_{k}$ is fully-supported with $\mathbb{P}^{m}$ probability one.

For each $k \in\{0, \ldots, \ell\}$, we denote by $x_{k}^{\star}(S)$ the (unique under Assumption 2) optimal solution of problem $P_{k}$. By Assumption 2, item $i i$ ), the size of the support set of $P_{k}$ is equal to $d$ with $\mathbb{P}^{m}$ probability one. Hence, for all $j \in$ $\{0, \ldots, \ell-1\}$, we define

$$
R_{j}(S)=\operatorname{supp}\left(x_{j}^{\star}(S)\right),
$$

which represents the support set at the $j$-th stage. Even though at the $\ell$-th stage of the proposed scheme no scenarios are discarded, we denote by $R_{\ell}(S)$ the support set associated to the optimal solution of $P_{\ell}$, i.e., we set $R_{\ell}(S)=$ $\operatorname{supp}\left(x_{\ell}^{\star}(S)\right)$. The final decision under this procedure is $x_{\ell}^{\star}(S)$, the solution for the last optimization problem.

To clarify the structure of the proposed removal scheme, we present in Figure 1 a diagram containing its main components. The scheme has $S$ scenarios as input and, at 


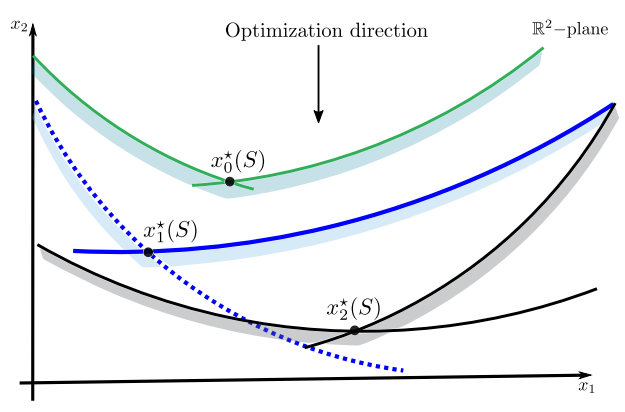

Fig. 2: Pictorial example that illustrates the scheme proposed in Figure 1. In this case, we have that $d=2, m=6, r=$ 4 , and $\ell=2$, and all the problems $P_{k}, k \in\{0,1,2\}$, satisfy Assumption 2, item ii). The objective function is given by $c^{\top} x=x_{2}$ (indicated by the downwards pointing arrow). The constraint sets are denoted by the different colors patterns: the green constraints are associated to the samples of $\operatorname{supp}\left(x_{0}^{\star}(S)\right)$, the blue ones to $\operatorname{supp}\left(x_{1}^{\star}(S)\right)$, and the black ones correspond to $\operatorname{supp}\left(x_{2}^{\star}(S)\right)$. Observe that the dashedblue constraint is removed by the scheme of Figure 1, but it is not violated by $x_{2}^{\star}(S)$.

each stage, the support set of the corresponding problem $P_{k}$ is removed. To further illustrate how the proposed scheme works, we consider the pictorial example of Figure 2. Note that $d=2, m=6$, and we remove $r=4$, thus requiring $\ell=2$ steps of the removal scheme of Figure 1. All the problems $P_{k}, k \in\{0,1,2\}$, are fully-supported, thus satisfying Assumption 2, item $i$ ). The objective function is given by $c^{\top} x=x_{2}$ and is indicated by the downwards pointing arrow. The corresponding solution for the intermediate problem is illustrated by $x_{k}^{\star}(S)$, for $k \in\{0,1,2\}$, and the support set of each stage by different colour patterns. For instance, the green constraints are the support set, namely, $\operatorname{supp}\left(x_{0}^{\star}(S)\right)$, of $P_{0}$. The shaded colour under each constraint corresponds to the region of the plane that violates that given constraint, e.g., we notice that $x_{1}^{\star}(S)$ violates both scenarios that belong to $\operatorname{supp}\left(x_{0}^{\star}(S)\right)$ and satisfies all the remaining ones.

\section{B. Main result}

We focus on the class of fully-supported problems. We are now in the position to present one of the main results of this paper. Consider the scheme of Figure 1 and let $x^{\star}(S)=x_{\ell}^{\star}(S)$. The next theorem provides guarantees for the probability of constraint violation associated to $x^{\star}(S)$. Note that the following result is not necessarily tight; in other words, fully-supportedness is not sufficient to guarantee a tight bound on the probability of constraint violation. It needs to be imposed in conjunction with another assumption (see Assumption 3 in the sequel), related to the violation properties of the removed scenarios. This is investigated in Section IV.

Theorem 4: Consider Assumption 2. Fix $\epsilon \in(0,1)$, set $r=$ $\ell d$ and let $m>r+d$. Consider also the scenario discarding scheme as encoded by (6) and illustrated in Figure 1, and let the minimizer of the $\ell$-th program be $x^{\star}(S)=x_{\ell}^{\star}(S)$. We then have that

$$
\begin{aligned}
\mathbb{P}^{m}\left\{\left(\delta_{1}, \ldots, \delta_{m}\right) \in \Delta^{m}\right. & \left.: \mathbb{P}\left\{\delta: g\left(x^{\star}(S), \delta\right)>0\right\}>\epsilon\right\} \\
& \leq \sum_{i=0}^{r+d-1}\left(\begin{array}{c}
m \\
i
\end{array}\right) \epsilon^{i}(1-\epsilon)^{m-i} .
\end{aligned}
$$

Before proceeding with the proof of Theorem 4, let us make some comments. Note that the first $\ell$-th iterations of the scheme in Figure 1 can be considered as a particular removal algorithm in the framework of [4]. Being so, we have proved a strictly better bound on the probability of violation that does not involve the combinatorial factor $\left(\begin{array}{c}r+d-1 \\ r\end{array}\right)$. Besides, for fully-supported problems, Theorem 4 relaxes the assumption in Theorem 2 that requires the optimal solution to violate all the constraints associated to the removed scenarios. Indeed, in the pictorial example of Figure 2, the dashed-blue constraint is removed at stage 1 , but it is not violated by the final solution of our scheme.

Proof: The proof is divided into four steps.

1) Definition of a suitable mapping: Consider Assumption 2. Let $m>(\ell+1) d$, and consider any set $C \subset S$, with $|C|=(\ell+1) d$. We consider the proposed scheme of Figure 1, fed by $C$ rather than $S$. All quantities introduced in this section depending on $S$ will now depend on $C$ instead. For a given set of indices $I \subset C$, we define

$$
\begin{aligned}
z^{\star}(I)=\underset{x \in X}{\operatorname{argmin}} c^{\top} x & \\
& \text { subject to } g(x, \delta) \leq 0, \text { for all } \delta \in I .
\end{aligned}
$$

Recall that $x_{k}^{\star}(C)$ denotes the minimizer of $P_{k}$ which in turn is based on the samples in $C \backslash \cup_{j=0}^{k-1} R_{j}(C)$, i.e., the ones that have not been removed up to stage $k$ of the proposed scheme. It thus holds that $x_{k}^{\star}(C)=z^{\star}\left(C \backslash \cup_{j=0}^{k-1} R_{j}(C)\right)$ - note that the argument of $z^{\star}$ in this case depends on $k, k \in\{0, \ldots, \ell\}$. Recall also that $R_{k}(C)=\operatorname{supp}\left(x_{k}^{\star}(C)\right)$.

Since we will be invoking the framework introduced in Section II-C, we define the mapping $\mathcal{A}: \Delta^{m} \rightarrow 2^{\Delta}$, with $\zeta=(\ell+1) d$, as

$$
\begin{aligned}
& \mathcal{A}(C)=\left\{\left\{\delta \in \Delta: g\left(x_{\ell}^{\star}(C), \delta\right) \leq 0\right\}\right. \\
& \cap\left\{\bigcap _ { k = 0 } ^ { \ell } \left\{\delta \in \Delta: c^{\top} z^{\star}(J \cup\{\delta\}) \leq c^{\top} x_{k}^{\star}(C),\right.\right. \\
& \left.\left.\left.\quad \text { for all } J \subset C \backslash \cup_{j=0}^{k-1} R_{j}(C), \text { with }|J|=d-1\right\}\right\}\right\} \\
& \cup\left\{\bigcup_{k=0}^{\ell-1} R_{k}(C)\right\}=\left(\mathcal{A}_{1}(C) \cap \mathcal{A}_{2}(C)\right) \cup \mathcal{A}_{3}(C) .
\end{aligned}
$$

The main motivation to define the mapping in (9) is the fact that its probability of violation will be shown to upper bound that of $\left\{\delta \in \Delta: g\left(x_{\ell}^{\star}(C), \delta\right) \leq 0\right\}$, which is ultimately the quantity we are interested in. 
Note that $\mathcal{A}(C)$ comprises three sets: (1) $\mathcal{A}_{1}(C)$ contains all realizations of $\delta$ for which the final decision of our proposed scheme $x_{\ell}^{\star}(C)=x^{\star}(C)$ remains feasible. This is the set whose probability of occurrence we are ultimately interested to bound; (2) $\mathcal{A}_{2}(C)$, the intersection of $\ell+1$ sets, indexed by $k \in\{0, \ldots, \ell\}$, each of them containing the realizations of $\delta$ such that, for all subsets of cardinality $d-1$ from the remaining samples at stage $k$, the cost $c^{\top} z^{\star}(J \cup\{\delta\})$ is lower than or equal to $c^{\top} x_{k}^{\star}(C)$. The former cost corresponds to appending $\delta$ to any set $J$ of $d-1$ scenarios from $C \backslash \cup_{j=0}^{k-1} R_{j}(C)$, while the latter corresponds to the cost of the minimizer $x_{k}^{\star}(C)$ of $P_{k}$. Informally, this inequality is of similar nature to that of the first set in $\mathcal{A}(C)$, however, rather than considering constraint satisfaction it only involves some cost dominance condition for each of the interim and the final optimal solutions. Observe that these sets carry information about the path taken by the proposed scheme, which is to be understood, in this context, as the sequence $\left(x_{k}(C)\right)_{k=0}^{\ell}$; (3) $\mathcal{A}_{3}(C)$, which includes all scenarios that are removed by the discarding scheme. Implicit in the definition of mapping (9) is the fact that, for any compression set $C$, all samples that are not removed in the intermediate stages must be contained in the set $\mathcal{A}_{1}(C) \cap \mathcal{A}_{2}(C)$.

2) An intermediate result: The following proposition establishes a basic property of any compression associated to the mapping (9).

Proposition 1: Consider Assumption 2. Set $r=\ell d$ and let $m>(\ell+1) d$. We have that $C \subset S$ is a compression set for $\mathcal{A}(C)$ in (9) if and only if, for all $k \in\{0, \ldots, \ell\}$,

$$
x_{k}^{\star}(C)=x_{k}^{\star}(S) .
$$

Proof: We first show necessity. Suppose that $C$ is a compression set but, for the sake of contradiction, we have that there exists $k \in\{0, \ldots, \ell\}$ and $\bar{\delta} \in S \backslash C$ such that

$$
x_{k}^{\star}(C) \neq x_{k}^{\star}(C \cup\{\bar{\delta}\}) .
$$

Let $\bar{k}$ be the minimum index such that (11) holds, while we have that $x_{j}^{\star}(C)=x_{j}^{\star}(C \cup\{\bar{\delta}\})$, for all $j<\bar{k}$. By Assumption 2, the last statement implies that $\operatorname{supp}\left(x_{j}^{\star}(C)\right)=$ $\operatorname{supp}\left(x_{j}^{\star}(C \cup\{\bar{\delta}\})\right)$, for all $j<\bar{k}$, as the support set of each optimal solution is unique. Hence, $R_{j}(C)=R_{j}(C \cup\{\bar{\delta}\})$ for all $j<\bar{k}$. By (8), we then have

$$
\begin{aligned}
x_{\bar{k}}^{\star}(C) & =z^{\star}\left(C \backslash \cup_{j=0}^{\bar{k}-1} R_{j}(C)\right), \\
x_{\bar{k}}^{\star}(C \cup\{\bar{\delta}\}) & =z^{\star}\left(\left(C \backslash \cup_{j=0}^{\bar{k}-1} R_{j}(C)\right) \cup\{\bar{\delta}\}\right) .
\end{aligned}
$$

Since the right-hand side of (13) involves one more scenario with respect to the right-hand side of (12), the feasible set of its corresponding optimization problem is smaller than that of (13). Moreover, by $x_{\bar{k}}^{\star}(C \cup\{\bar{\delta}\}) \neq x_{\bar{k}}^{\star}(C)$ and Assumption 2 we obtain that $\delta$ belongs to the support set of $x_{\bar{k}}^{\star}(C \cup\{\bar{\delta}\})$, as its removal results in a different optimal solution with lower cost.

In other words, there exists $\bar{J} \subset C \backslash \cup_{j=0}^{\bar{k}-1} R_{j}(C)$ (in fact, $\left.\bar{J}=\operatorname{supp}\left(x_{\bar{k}}^{\star}(C \cup\{\bar{\delta}\})\right) \backslash\{\bar{\delta}\}\right)$ of cardinality $d-1$ such that by (13), we have that

$$
c^{\top} x_{\bar{k}}^{\star}(C)<c^{\top} x_{\bar{k}}^{\star}(C \cup\{\bar{\delta}\})=c^{\top} z^{\star}(\bar{J} \cup\{\bar{\delta}\}) .
$$

However, this is a contradiction, as (14) implies that $\bar{\delta} \notin$ $\mathcal{A}_{2}(C)$ and $C$ is assumed to be a compression. This shows that $x_{k}^{\star}(C)=x_{k}^{\star}(C \cup\{\delta\})$, for any $\delta \in S \backslash C$, and any $k \in\{0, \ldots, \ell\}$. Proceeding inductively, adding one by one each element in $S \backslash C$, we can show that $x_{k}^{\star}(C)=x_{k}^{\star}(S)$, for any $k \in\{0, \ldots, \ell\}$, thus concluding the necessity proof.

We now show sufficiency. Let $C \subset S$ be such that $x_{k}^{\star}(C)=$ $x_{k}^{\star}(S)$ for all $k \in\{0, \ldots, \ell\}$. Recalling the definition of the mapping $\mathcal{A}(C)$ from (9) we note that, under this scenario, the sets $\mathcal{A}_{1}(C)$ and $\mathcal{A}_{3}(C)$ are trivially equal to $\mathcal{A}_{1}(S)$ and $\mathcal{A}_{3}(S)$, respectively. Moreover, since $C \subset S$ and $x_{k}^{\star}(C)=$ $x_{k}^{\star}(S)$ for all $k \in\{0, \ldots, \ell\}$, which implies that $R_{k}(C)=$ $R_{k}(S)$ by Assumption 2, we have that $S \backslash \cup_{j=0}^{k-1} R_{j}(S)=$ $S \backslash \cup_{j=0}^{k-1} R_{j}(C) \supset C \backslash \cup_{j=0}^{k-1} R_{j}(C)$. The latter implies that $\mathcal{A}_{2}(S) \subset \mathcal{A}_{2}(C)$. By construction we have that $\delta \in \mathcal{A}(S)$ for all $\delta \in S$. This in turn implies that if a sample is not removed, then it will have to be included in $\mathcal{A}_{2}(S)$, and due to the established inclusion also in $\mathcal{A}_{2}(C)$. Since $\mathcal{A}_{1}(S)=\mathcal{A}_{1}(C)$ and $\mathcal{A}_{3}(S)=\mathcal{A}_{3}(C)$, we then have that $\delta \in \mathcal{A}(C)$ for all $\delta \in S$, showing that $C$ is a compression set. This concludes the proof of the proposition.

3) Existence and uniqueness of a compression: A natural compression candidate is

$$
C=\bigcup_{k=0}^{\ell} \operatorname{supp}\left(x_{k}^{\star}(S)\right),
$$

as it consists of the support samples for $P_{k}, k \in\{0, \ldots, \ell\}$.

Existence: We prove that $C$ in (15) is a compression set. By the sufficient part of Proposition 1, it suffices to show that the set $C$ in (15) satisfies $x_{k}^{\star}(C)=x_{k}^{\star}(S)$, for all $k \in\{0, \ldots, \ell\}$. We will show this by means of induction. For the base case $k=0$, notice that

$$
c^{\top} x_{0}^{\star}(S)=c^{\top} z^{\star}(S)=c^{\top} z^{\star}\left(\operatorname{supp}\left(x_{0}^{\star}(S)\right)\right)=c^{\top} x_{0}^{\star}(C),
$$

where the first equality is due to (8), the second equality is due to the fact that $\operatorname{supp}\left(x_{0}^{\star}(S)\right)$ is the support set of $x_{0}^{\star}(S)$, while the last equality is due to Assumption 2, the definition of support set and the fact that $\operatorname{supp}\left(x_{0}^{\star}(S)\right) \subset C$. From the above relation and Assumption 2, we conclude that $x_{0}^{\star}(C)=x_{0}^{\star}(S)$.

To complete the induction argument, we assume that $x_{j}^{\star}(C)=x_{j}^{\star}(S)$ for all $j \in\{0, \ldots, \bar{k}\}$, for some $\bar{k}<\ell$. We will show that $x_{\bar{k}+1}^{\star}(C)=x_{\bar{k}+1}^{\star}(S)$. To this end, by Assumption $2, x_{j}^{\star}(C)=x_{j}^{\star}(S)$ for all $j \leq \bar{k}$ implies that $\operatorname{supp}\left(x_{j}^{\star}(C)\right)=\operatorname{supp}\left(x_{j}^{\star}(S)\right)$, for all $j \leq \bar{k}$, as the support set of each optimal solution is unique. Moreover, $R_{j}(C)=R_{j}(S)$ for all $j<\bar{k}$, as $R_{j}(C)=\operatorname{supp}\left(x_{j}^{\star}(C)\right)$ for fully-supported problems. Similarly to the base case we 
have that

$$
\begin{aligned}
c^{\top} x_{\bar{k}+1}^{\star}(C) & =c^{\top} z^{\star}\left(C \backslash \cup_{j=0}^{\bar{k}} R_{j}(S)\right) \\
& \leq c^{\top} z^{\star}\left(S \backslash \cup_{j=0}^{\bar{k}} R_{j}(S)\right)=c^{\top} x_{\bar{k}+1}^{\star}(S),
\end{aligned}
$$

where the first and last equalities are due to (8), and the inequality is due to the fact that $C \backslash \cup_{j=0}^{\bar{k}} R_{j}(S) \subseteq S \backslash$ $\cup_{j=0}^{\bar{k}} R_{j}(S)$. Moreover, since $C \subset S$ and $R_{j}(C)=R_{j}(S)$ for all $j<\bar{k}$, we have that $c^{\top} x_{\bar{k}+1}^{\star}(S) \leq c^{\top} x_{\bar{k}+1}^{\star}(C)$. This implies that $x_{\bar{k}+1}^{\star}(C)=x_{\bar{k}+1}^{\star}(S)$, thus concluding the induction proof. In other words, we have shown that

$$
x_{k}^{\star}(C)=x_{k}^{\star}(S), \text { for all } k \in\{0, \ldots, \ell\} .
$$

Relation (17) and the sufficiency part of Proposition 1 show that the candidate $C$ in (15) is a compression set.

Uniqueness: To show that $C$ in (15) is the unique compression set, let $C^{\prime}$ be another compression set of size $(\ell+1) d$. We show that $C^{\prime}=C$. Since $C^{\prime} \subset S$ is a compression, Proposition 1 (necessity part) implies that $x_{k}^{\star}\left(C^{\prime}\right)=x_{k}^{\star}(S)$, for all $k \in\{0, \ldots, \ell\}$, as $C^{\prime}$ is a compression. Hence, we have that $x_{k}^{\star}(C)=x_{k}^{\star}\left(C^{\prime}\right)$ for all $k \in\{0, \ldots, \ell\}$, thus showing that $C=C^{\prime}$ by Assumption 2. This concludes the uniqueness part.

4) Linking Theorem 3 with the probability of constraint violation: Recall that

$$
\mathcal{A}(C)=\left(\mathcal{A}_{1}(C) \cap \mathcal{A}_{2}(C)\right) \cup \mathcal{A}_{3}(C),
$$

where the individual sets are as in (9). Recall also that $\mathcal{A}_{3}(S)$ is a discrete set. Let $C \subset S$ with $|C|=(\ell+1) d$ be the unique compression defined in (15). We have that

$$
\begin{aligned}
\mathbb{P} & \{\mathcal{A}(C)\}=\mathbb{P}\left\{\mathcal{A}_{1}(C) \cap \mathcal{A}_{2}(C)\right\}, \\
& \leq \mathbb{P}\left\{\mathcal{A}_{1}(C)\right\}=\mathbb{P}\left\{\delta \in \Delta: g\left(x^{\star}(C), \delta\right) \leq 0\right\}, \\
& =\mathbb{P}\left\{\delta \in \Delta: g\left(x^{\star}(S), \delta\right) \leq 0\right\},
\end{aligned}
$$

where the first equality is due to the fact that $\mathbb{P}\left\{\mathcal{A}_{3}(C)\right\}=0$, since $\mathcal{A}_{3}(C)$ is a discrete set and $\mathbb{P}$ is non-atomic, which prevents scenarios to have accumulation points with nonzero probability, while the inequality is due to the fact that $\mathcal{A}_{1}(C) \cap \mathcal{A}_{2}(C) \subseteq \mathcal{A}_{1}(C)$. The second to last equality is by definition of $\mathcal{A}_{1}(C)$, and the last one follows from the fact that $x^{\star}(C)=x^{\star}(S)$ (see (17)).

We then have that if $\mathbb{P}\left\{\delta \in \Delta: g\left(x^{\star}(S), \delta\right)>0\right\}>\epsilon$ then $\mathbb{P}\{\delta \in \Delta: \delta \notin \mathcal{A}(C)\}>\epsilon$. As a result, $\left\{\left(\delta_{1}, \ldots, \delta_{m}\right) \in\right.$ $\left.\Delta^{m}: \mathbb{P}\left\{\delta \in \Delta: g\left(x^{\star}(S), \delta\right)>0\right\}>\epsilon\right\} \subseteq\left\{\left(\delta_{1}, \ldots, \delta_{m}\right) \in\right.$ $\left.\Delta^{m}: \mathbb{P}\{\delta \in \Delta: \delta \notin \mathcal{A}(C)\}>\epsilon\right\}$. The last statement implies then that

$$
\mathbb{P}^{m}\left\{\left(\delta_{1}, \ldots, \delta_{m}\right) \in \Delta^{m}: \mathbb{P}\left\{\delta \in \Delta: g\left(x^{\star}(S), \delta\right)>0\right\}>\epsilon\right\}
$$$$
\leq \mathbb{P}^{m}\left\{\left(\delta_{1}, \ldots, \delta_{m}\right) \in \Delta^{m}: \mathbb{P}\{\delta \notin \mathcal{A}(C)\}>\epsilon\right\} .
$$

Therefore, since the set $C$ in (15) is the unique compression of $\mathcal{A}(C)$, by Theorem 3 we have that

$$
\begin{aligned}
\mathbb{P}^{m}\left\{\left(\delta_{1}, \ldots, \delta_{m}\right) \in \Delta^{m}\right. & : \mathbb{P}\{\delta \in \Delta: \delta \notin \mathcal{A}(C)\}>\epsilon\} \\
\leq & \sum_{i=0}^{r+d-1}\left(\begin{array}{c}
m \\
i
\end{array}\right) \epsilon^{i}(1-\epsilon)^{m-i} .
\end{aligned}
$$

By (20) and (21), we obtain the result. This concludes the proof of Theorem 4.

\section{TIGHTNESS OF THE BOUND OF THEOREM 4}

\section{A. Class of programs for which the bound is tight}

We provide a sufficient condition on the problems $P_{k}$ so that the solution returned by the scheme of Figure 1 achieves the upper bound given by the right-hand side of (7) when all the intermediate problems $P_{k}, k=0, \ldots, \ell$, are fully-supported. The result of this section implies that the bound of Theorem 4 is tight, i.e., there exists a class of convex scenario programs where it holds with equality.

To this end, we replace the mapping $\mathcal{A}$ in (9) with $\overline{\mathcal{A}}: \Delta^{m} \rightarrow$ $2^{\Delta}$ defined by

$$
\begin{aligned}
\overline{\mathcal{A}}(C)=\left\{\delta \in \Delta: g\left(x_{\ell}^{\star}(C), \delta\right) \leq 0\right\} \cup & \\
& \left\{\bigcup_{k=0}^{\ell-1} \operatorname{supp}\left(x_{k}^{\star}(C)\right)\right\} .
\end{aligned}
$$

Note that $\overline{\mathcal{A}}(C)$ coincides with the one in (18), but without the set $\mathcal{A}_{2}(C)$ in its definition. We impose the following assumption.

Assumption 3: Fix any $S=\left\{\delta_{1}, \ldots, \delta_{m}\right\} \in \Delta^{m}$ and let $C \subset S$. For any $k \in\{0, \ldots, \ell\}$ and $\delta \in S$ such that $\delta \in$ $\operatorname{supp}\left(x_{k}^{\star}(C)\right)$, we have that

$$
g\left(z^{\star}(J), \delta\right)>0
$$

for all $J \subset C \backslash\left(\cup_{j=0}^{k-1} \operatorname{supp}\left(x_{j}^{\star}(C)\right) \cup\{\delta\}\right)$ with $|J|=d$.

Assumption 3 imposes a restriction on the class of fullysupported problems. For instance, the pictorial example of Figure 2 does not satisfy Assumption 3, even though all the intermediate problems $P_{k}$ are fully-supported, as the dashedblue removed constraint is not violated by the resulting solution. Indeed, Assumption 3 requires that, with $\mathbb{P}^{m}$ probability, whenever a sample belongs to the support scenarios of any intermediate problem, then the scenario associated with it is violated by all the solutions that could have been obtained using any subset of cardinality $d$ from the remaining samples. Note that verifying Assumption 4 is hard in general; we show in the next subsection an example that satisfies this requirement and admits an analytic solution. Assumption 3 is similar to the requirement of Theorem 2 [4], [5], however, in Theorem 5 below we exploit it in conjunction with the discarding scheme of Figure 1 to show that the result of Theorem 4 is tight. This serves as a constructive argument for the existential result of [4].

Note that in this paper we do not offer any means to check the validity of Assumption 3; however, we show that this class of problems is not empty in the next section.

Theorem 5: Consider Assumptions 2 and 3. Fix $\epsilon \in(0,1)$, set $r=\ell d$ and let $m>r+d$. Consider also the scenario discarding scheme as encoded by (6) and illustrated in Figure 
1 , and let the minimizer of the $\ell$-th program be $x^{\star}(S)=$ $x_{\ell}^{\star}(S)$. We then have that

$$
\begin{aligned}
\mathbb{P}^{m}\left\{\left(\delta_{1}, \ldots, \delta_{m}\right) \in \Delta^{m}\right. & \left.: \mathbb{P}\left\{\delta: g\left(x^{\star}(S), \delta\right)>0\right\}>\epsilon\right\} \\
& =\sum_{i=0}^{r+d-1}\left(\begin{array}{c}
m \\
i
\end{array}\right) \epsilon^{i}(1-\epsilon)^{m-i} .
\end{aligned}
$$
[17].

Proof: Omitted for brevity. This proof can be found in

\section{B. An example with an analytic solution}

We revisit the simple problem studied in [4], [18] and show that it satisfies Assumption 3. We compute analytically the violation probability of the solution returned by applying the scheme of Figure 1 to this problem and show that the resulting violation probability coincides with the result of Theorem 5.

To this end, fix $m \in \mathbb{N}$ and $r<m$, and consider the procedure of Section III-A, which involves a sequence of $\ell+1$ problems. For $k=0, \ldots, \ell$, each of them in the form of $P_{k}$, given by

$$
\begin{aligned}
\underset{x \in[0,1]}{\operatorname{minimize}} & x \\
\text { subject to } & x \geq \delta_{i}, \quad i \in S \backslash \cup_{j=0}^{k-1} R_{j}(S) .
\end{aligned}
$$

We further assume that all samples are extracted from a uniform distribution over the interval $[0,1]$. Note that (24) satisfies Assumption 2. Also notice that Assumption 4 is satisfied for this problem, as $x_{k}^{\star}=\max _{i \in S \backslash \cup_{j=0}^{k-1} R_{j}(S)} \delta_{i}$, i.e., the maximum among the scenarios available at stage $k$ of the discarding process. Under the choice of a uniform distribution, the support set is a singleton, i.e., the maximizing scenario is unique, with $\mathbb{P}^{m}$-probability one. Therefore, once the single support scenario is removed, the new minimizer will necessarily be lower thus violating the removed scenario. Note that since this is a one-dimensional problem $(d=1)$, our procedure involves removing samples one by one.

Let $\epsilon \in(0,1)$ and $r=\ell d=\ell<m$ (since this is an onedimensional problem), and consider the set:

$$
B=\mathbb{P}^{m}\left\{\left(\delta_{1}, \ldots, \delta_{m}\right) \in \Delta^{m}: x^{\star}(S)<1-\epsilon\right\},
$$

which represents the measure of samples such that the probability of constraint violation is greater that $\epsilon$, as $\mathbb{P}\{\delta \in$ $\left.\Delta: x^{\star}(S)<\delta\right\}=1-x^{\star}(S)$ due to the fact that $\mathbb{P}$ is uniform on $[0,1]$. Moreover,

$$
\begin{gathered}
A_{0}=\mathbb{P}^{m}\left\{\left(\delta_{1}, \ldots, \delta_{m}\right) \in \Delta^{m}: \text { for all } i=1, \ldots, m,\right. \\
\left.\delta_{i} \leq 1-\epsilon\right\},
\end{gathered}
$$

and for all $\ell=1, \ldots, m$,

$$
\begin{array}{r}
A_{i}=\mathbb{P}^{m}\left\{\left(\delta_{1}, \ldots, \delta_{m}\right)\right. \\
\qquad \Delta^{m}: \text { there exist exactly } \\
i \text { samples greater than } 1-\epsilon\} .
\end{array}
$$

Observe that $\left\{A_{i}\right\}_{i=0}^{m}$ forms a partition of $\Delta^{m}$, and notice that

$$
\begin{gathered}
\mathbb{P}^{m}\left\{A_{0}\right\}=\mathbb{P}^{m}\left\{\left(\delta_{1}, \ldots, \delta_{m}\right) \in \Delta^{m}: \delta_{i} \leq 1-\epsilon,\right. \text { for all } \\
i=1, \ldots, m\}=(1-\epsilon)^{m},
\end{gathered}
$$

where the last equality is due to sample independence and the fact that $\mathbb{P}^{m}\{\delta \in \Delta: \delta>1-\epsilon\}=\epsilon$ (due to the fact that $\mathbb{P}$ is uniform on $[0,1]$ ). Since $A_{i}$ involves $i$ independent samples, we also have that

$$
\mathbb{P}^{m}\left\{A_{i}\right\}=\left(\begin{array}{c}
m \\
i
\end{array}\right) \epsilon^{i},
$$

where the factor $\left(\begin{array}{c}m \\ i\end{array}\right)$ accounts for all combinations of $i$ out of $m$ samples. Moreover, for $i \leq r, \mathbb{P}^{m}\left\{B \mid A_{i}\right\}=(1-\epsilon)^{m-i}$ since it involves conditioning on exactly $i$ samples being removed, and computing the probability that the returned solution $x^{\star}(S)$ is feasible for the remaining $m-i$ samples. By the total law of probability, we then have that

$$
\begin{aligned}
& \mathbb{P}^{m}\left\{\left(\delta_{1}, \ldots, \delta_{m}\right) \in \Delta^{m}: x^{\star}(S)<1-\epsilon\right\}=\mathbb{P}^{m}\{B\} \\
& =\sum_{i=0}^{m} \mathbb{P}^{m}\left\{A_{i} \cap B\right\} \\
& =\sum_{i=0}^{r} \mathbb{P}^{m}\left\{A_{i} \cap B\right\}+\sum_{i=r+1}^{m} \mathbb{P}^{m}\left\{A_{i} \cap B\right\} .
\end{aligned}
$$

The terms involving $i>r$ are zero because $\left(\delta_{1}, \ldots, \delta_{m}\right) \in B$ implies that at most $r$ of samples are greater than $1-\epsilon$, i.e., $\mathbb{P}^{m}\left\{B \mid A_{i}\right\}=0$, for all $i>r$. Hence,

$$
\begin{aligned}
& \mathbb{P}^{m}\left\{\left(\delta_{1}, \ldots, \delta_{m}\right) \in \Delta^{m}: x^{\star}(S)<1-\epsilon\right\} \\
& =\sum_{i=0}^{r} \mathbb{P}^{m}\left\{A_{i} \cap B\right\}=\sum_{i=0}^{r} \mathbb{P}^{m}\left\{B \mid A_{i}\right\} \mathbb{P}^{m}\left\{A_{i}\right\} \\
& =\sum_{i=0}^{r}\left(\begin{array}{c}
m \\
i
\end{array}\right) \epsilon^{i}(1-\epsilon)^{m-i},
\end{aligned}
$$

where the last equality follows from (28) and (29). Note that this result coincides with the bound of Theorem 5 .

\section{Conclusion}

In this paper, we revisited the sampling and discarding approach within the scenario approach theory and derived a tight bound on the probability of constraint violation for the obtained solution. To this end, we analyzed a scheme to remove constraints that is composed of a cascade of scenario programs, where at each stage a subset of scenarios related to the support set of the associated optimal solution is removed. Our tight results refer to this particular discarding scheme and the class of fully-supported programs.

Current work involves showing that the proposed bound is valid, albeit not tight, for any convex scenario program that is not necessarily fully-supported. Under a mild nondegeneracy assumption we have established such a result in [17].

Our present analysis is limited to cases where the number of removed scenarios is a multiple of the dimension of the 
optimization problem. Current work concentrates towards relaxing this requirement, and investigating the effect in the performance of discarding schemes based on the dual variables of the associated constraints.

\section{ACKNOWLEDGEMENT}

The authors are grateful to Professors Simone Garatti and Marco Campi for extended discussions on the results presented in this paper. Special thanks to Prof. Garatti for several insightful technical comments.

\section{REFERENCES}

[1] G. Calafiore and M. C. Campi, "Uncertain convex programs: Randomized solutions and confidence levels," Mathematical Programming, vol. 102 , no. 1 , pp. $25-46,2005$

[2] M. C. Campi and S. Garatti, "The exact feasibility of randomized solutions of uncertain convex programs," SIAM Journal on Optimization, vol. 19 , no. 3, pp. 1211-1230, 2008.

[3] M. Campi and S. Garatti, Introduction to the Scenario Approach MOS-SIAM Series on Optimization, 2018.

[4] _ "A Sampling-and-Discarding Approach to Chance-Constrained Optimization: Feasibility and Optimality," J Optim Theory Appl, vol. 148, pp. 257-280, 2011

[5] G. C. Calafiore, "Random convex programs," SIAM Journal on Optimization, vol. 20 , no. 6 , pp. 3427-3464, 2010

[6] S. Grammatico, X. Zhang, K. Margellos, P. Goulart, and J. Lygeros, "A Scenario Approach for Non-Convex Control Design," IEEE Trans actions on Automatic Control, vol. 61, no. 2, pp. 334-345, 2016.

[7] G. C. Calafiore, "Repetitive Scenario Design," IEEE Transactions on Automatic Control, vol. 62, no. 3, pp. 1125-1137, 2017.
[8] M. C. Campi and S. Garatti, "Wait-and-judge scenario optimization," Mathematical Programming, vol. 167, no. 1, pp. 155-189, 2018.

[9] M. C. Campi, S. Garatti, and F. A. Ramponi, "A General Scenario Theory for Nonconvex Optimization and Decision Making," IEEE Transactions on Automatic Control, vol. 63, no. 12, pp. 4067-4078, 2018.

[10] S. Garatti and M. C. Campi, "Complexity-based modulation of the data-set in scenario optimization," European Control Conference, no. 2, pp. 1386-1391, 2019.

[11] D. Paccagnan and M. C. Campi, "The scenario approach meets uncertain variational inequalities and game theory," in 58th Conference on Decision and Control, Nice, France, 2019, pp. 6124-6129.

[12] F. Fele and K. Margellos, "Probabilistic sensitivity of Nash equilibria in multi-agent games: a wait-and-judge approach," in 58th Conference on Decision and Control, Nice, France, 2019, pp. 5026-5031.

[13] _ , "Probably Approximately Correct Nash Equilibrium Learning," arXiv:1903.10387 [math.OC], pp. 1-12, 2019

[14] M. Vidyasagar, Learning and Generalization. Springer, 2002.

[15] K. Margellos, M. Prandini, and J. Lygeros, "On the connection between compression learning and scenario based single-stage and cascading optimization problems," IEEE Transactions on Automatic Control, vol. 60, no. 10, pp. 2716-2721, 2015.

[16] S. Floyd and M. Warmuth, "Sample compression, learnability, and the Vapnik-Chervonenkis dimension," Machine Learning, no. 21, pp. 269-304, 1995.

[17] L. Romao, K. Margellos, and A. Papachristodoulou, "On the exact feasibility of convex scenario programs with discarded constraints," arXiv:2003.13433 [math.OC], pp. 1-15, 2020.

[18] M. C. Campi and G. C. Calafiore, "Notes on the scenario design approach," IEEE Transactions on Automatic Control, vol. 54, no. 2, pp. $382-385,2009$. 